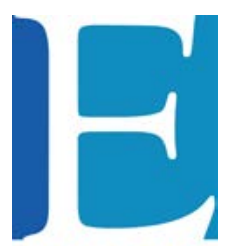

EVALUAR
2017, Vol. 17, No. 1

ISSN $1667-4545$

Recuperado de www.revistas.unc.edu.ar

\title{
Estandarización del Child Behavior Checklist para preescolares de población urbana de Argentina
}

\author{
Natalia Vázquez * 1, Virginia Corina Samaniego ${ }^{2}$ \\ 1. Consejo Nacional de Investigaciones Científicas y Técnicas (CONICET).Centro de Investigaciones en Psicología y \\ Psicopedagogía. Facultad de Psicología y Psicopedagogía, Pontificia Universidad Católica Argentina, Argentina. \\ 2. Centro de Investigaciones en Psicología y Psicopedagogía. Facultad de Psicología y Psicopedagogía, Pontificia \\ Universidad Católica Argentina, Argentina. \\ Introducción \\ Método \\ Resultados \\ Discusión \\ Referencias
}

Recibido: 22/02/20176 Revisado: 02/03/2017 Aceptado: 14/03/2017

\section{Resumen}

La presencia de problemas en la salud mental de un niño puede afectar sus posibilidades de desarrollar al máximo sus recursos emocionales y cognitivos. El diagnóstico precoz de estos problemas resulta una medida preventiva por excelencia, por ello es necesario contar con instrumentos validados. Se propuso estandarizar el Child Behavior Checklist (CBCL 1 1 $12-5$ ) en su versión en español para padres. Se realizó un estudio instrumental, sobre una muestra de 541 niños en edad preescolar pertenecientes a la población urbana de Argentina, $n=369$ pertenecientes a la población general, y $\mathrm{n}=172$ de un grupo clínico de salud mental. Se demostró que el CBCL 1 1/2-5 permite evaluar grados de problemas comportamentales y emocionales en edades tempranas. Las escalas generales (puntaje total, problemas externalizantes y problemas internalizantes) discriminaron adecuadamente entre el grupo clínico y el grupo de población general. Se corroboró la confiabilidad del instrumento a partir del alto grado de acuerdo entre informantes (madre y padre), los valores adecuados de consistencia interna y los altos valores de correlación pasados 7 días (test-retest) y pasados 6 meses (estabilidad). La estandarización del CBCL 1 1/2-5 permite contar con un instrumento de detección temprana de problemas comportamentales y emocionales, fortaleciendo la capacidad diagnóstica de problemas en la salud mental infantil.

\begin{abstract}
Mental health problems in children can affect their ability to develop emotional and cognitive resources. An early diagnosis of these problems is an excellent prevention strategy; therefore, it is necessary to have validated instruments. We proposed to standardize the Child Behavior Checklist (CBCL 1 $1 \frac{12-5}{2}$ ) in its Spanish version for parents. A methodological study was carried out on a sample of 541 preschool children from urban population of Argentina, $n=369$ from the general population and $n=$ 172 from a mental health clinical group. According to the results, the CBCL $1 \frac{1}{2}-5$ is appropriate to evaluate degrees of behavioral and emotional problems in early ages. The broad scales (total score, externalizing problems and internalizing problems) adequately discriminated between the clinical group and the general population group. The reliability of the instrument was corroborated by high degree of agreement between informants (parents), adequate values of internal consistency, high correlation values after 7 days (test-retest), and after 6 months (stability). The standarized CBCL 11/2-5 allows an early detection of behavioral and emotional problems, improving the diagnosis of mental health problems in children.
\end{abstract}

Keywords: children, mental health, prevention, $C B C L$

Palabras clave: niños, salud mental, prevención, $C B C L$

\footnotetext{
*Correspondencia a: Natalia Vázquez, Av. Alicia Moreau de Justo 1300, C1107AAZ, Buenos Aires, Argentina. Tel.: 4349-0200 (int. 1107). natalia_vazquez@uca.edu.ar

Cómo citar este artículo: Vázquez, N., \& Samaniego, V. C. (2017). Estandarización del Child Behavior Checklist para preescolares de población urbana de Argentina. Revista Evaluar, 17(1), 65-79. Recuperado de https://revistas.unc.edu.ar/index.php/revaluar

Nota: Esta investigación fue realizada en el marco de dos Becas de Postgrado de CONICET (Tipo I y de finalización de doctorado) otorgadas a la Dra. Natalia Vázquez.

Agradecimiento: Se agradece a los jardines de infantes y maternales de capital federal y de la ciudad de Mercedes, Buenos Aires, que brindaron su espacio para el desarrollo de la investigación. A todos los padres y madres que generosa y desinteresadamente han participado del estudio.
} 


\section{Introducción}

Uno de los grandes temas de estudio de la psicología, a nivel mundial y a lo largo de la historia, ha sido la diferencia entre una adecuada salud mental y la presencia de psicopatología, considerando las implicancias de esta última en el contexto cotidiano de quien la padece. La presencia de problemas en la salud mental de un niño puede afectar sus posibilidades de desarrollar vínculos sanos, adquirir habilidades cognoscitivas y competencias emocionales. La capacidad de detectar tempranamente estos problemas, con instrumentos que resulten válidos y confiables, posibilitará el inicio de un proceso psicoterapéutico que mejore el pronóstico del niño. El diagnóstico precoz de estos problemas en edades tempranas se convierte en una medida preventiva por excelencia, ya que reduce la aparición de trastornos que podrían a futuro afectar el aprendizaje en la edad escolar o generar discapacidad a largo plazo (Achenbach \& Rescorla, 2000; Martinez, 2009; Pihlakoski et al., 2006).

Los niños pequeños suelen ser llevados por sus padres a una consulta clínica porque los observan agresivos, desafiantes, hiperactivos, o con dificultades para prestar atención; pero estos comportamientos en la edad preescolar son también típicos del desarrollo. Uno de los parámetros que nos permite discriminar si las dificultades que perciben los padres son significativas como para requerir atención clínica son los datos epidemiológicos basados en el contexto sociocultural del niño; esto implica conocer la prevalencia de problemas en la salud mental infantil (Rescorla et al., 2011). Sin embargo, se advierte que aún existen muchas limitaciones en la conceptualización, definición, medición y estudio de los problemas comportamentales $y$ emocionales en niños pequeños de edad preescolar. Entre ellas pueden mencionarse: a) la falta de consenso para determinar qué afectos o comportamientos son normales o patológicos para esta edad; b) la poca confiabilidad de la información que los padres brindan sobre el comportamiento de sus hijos, y las dificultades para combinar información de múltiples informantes; c) las limitaciones en la conceptualización y medición de los trastornos psicopatológicos; d) la escasez de estudios sobre los factores de riesgo asociados, y e) la poca cantidad de artículos, a nivel mundial, que indiquen la prevalencia, distribución y evolución de los problemas en la salud mental infantil (Dougherty et al., 2015; Egger \& Angold, 2006; Skovgaard, Houmann, Landorph, \& Christiansen, 2004; Skovgaard, Christiansen, Houmann, Landorph, \& Jørgensen, 2008). En la producción científica de Argentina se advierte que también existen lagunas en el conocimiento epidemiológico sobre la salud mental de los más pequeños. Se reconocen como antecedentes los estudios realizados por Samaniego en niños de edad escolar con el CBCL, en población general y en población clínica (Samaniego, 2004, 2005, 2008, 2012, 2015; Slapak, Cervone, Luzzi, \& Samaniego, 2002); mientras que existe también un antecedente de validación de otro cuestionario de evaluación sobre el comportamiento infantil realizado por Molina y colaboradores (Molina, Calero, \& Raimundi, 2014). En preescolares, la cantidad de estudios es menor. Una de las investigaciones encontró que el $24 \%$ de las admisiones realizadas durante un año en un servicio de salud mental infanto-juvenil perteneciente a un hospital general, se correspondían con esta franja etaria (Schu, Zan, \& Vázquez, 2011). Un segundo estudio, en una muestra de población general, aportó evidencia que sostiene que a mayor nivel de problemas comportamentales y emocionales en los padres se observa mayor nivel de estos mismos problemas en sus hijos (Rivas, Vázquez, \& Samaniego, 2011).

Tanto para la actividad clínica como para la epidemiológica, resulta esencial contar con instrumentos de evaluación cuyas propiedades psicométricas hayan sido evaluadas en el con- 
texto cultural en el que se emplearán. Dentro de las escalas del sistema ASEBA (Achenbach System of Empirically Based Assessment), el Child Behavior Checklist (CBCL 1 1/2-5) es un instrumento para niños de entre 1 año y medio y 5 años de edad, que surge como revisión de una versión anterior que era solamente para niños de 2 y 3 años, el CBCL/2-3 (Achenbach \& Rescorla, 2000; Achenbach, 1992). El CBCL $1 \frac{1}{2}$-5 ha sido aplicado en al menos 24 sociedades distintas $(\mathrm{N}=19,850)$ y ha demostrado ser una herramienta válida y confiable para medir problemas comportamentales y emocionales en niños pequeños. Siendo una de las escalas con mayor uso transcultural, cuenta hasta el momento con la más grande y diversa base de datos para comparar los niveles de problemas en esta edad (Ivanova et al., 2010; Liu, Leung, Sun, Li, \& Liu, 2012; Rescorla et al., 2011). El cuestionario emplea un lenguaje sencillo para describir el funcionamiento de niños pequeños en diversas condiciones; lo cual facilita su uso en diferentes contextos: en la práctica clínica, en ámbitos de educación, en contextos médicos, en procesos de adopción y en el ámbito forense. También puede señalarse como ventaja que su administración resulta económica, es autoadministrable y requiere un tiempo de respuesta breve de aproximadamente 15 minutos, al tiempo que tiene gran aceptación por parte de los adultos que informan sobre el niño (Achenbach, 1992; Achenbach \& Rescorla, 2000, 2004; Achenbach, Rescorla, \& Maruish, 2004; Rescorla, 2005). Este último aspecto es importante dado que los niños no tienen la posibilidad de discriminar un problema y demandar atención por él, sino que son sus padres o adultos responsables quienes deben ser capaces de percibir los problemas e identificarlos como tales, lo más tempranamente posible, lo que les permitirá actuar para prevenir futuros problemas en el desarrollo.

Dentro de los principales antecedentes que demuestran el uso del CBCL 1 1/2-5 a ni- vel mundial, puede destacarse su utilidad para identificar a niños pequeños en riesgo de tener un cuadro de autismo. Esto permite reducir la brecha de tiempo que usualmente existe entre las primeras preocupaciones de los padres y el diagnóstico de un trastorno del espectro autista; y ofrece al niño la oportunidad de una intervención terapéutica específica y temprana que mejore su pronóstico (Limberg, Gruber, \& Noterdaeme, 2016; Muratori et al., 2011; Pandolfi, Magyar, \& Dill, 2009; Predescu, Şipos, Dobrean, \& Miclutia, 2013). El instrumento se ha aplicado también en estudios para demostrar la eficacia de modelos de tratamiento clínico para niños pequeños (Müller et al., 2015) o, por ejemplo, para el estudio de factores de riesgo prenatales, como el consumo de tabaco por parte de la madre durante el embarazo (Liu, Leung, McCauley, Ai, \& Pinto-Martin, 2013), o postnatales, como la presencia de elevados valores de plomo en la sangre del niño (Liu et al., 2014). También es importante mencionar su uso en Latinoamérica. Un estudio en Chile demostró que el CBCL 1 1/2-5 es un instrumento válido y confiable para ser aplicado en ese contexto (Lecannelier et al., 2014). Otro trabajo en Brasil ha encontrado que altos niveles de control psicológico materno de tipo crítico se asocian con problemas en la dimensión ansioso/depresiva y problemas de retraimiento en niños de edad preescolar (Lins \& Alvarenga, 2015).

Considerando que se habían demostrado propiedades psicométricas adecuadas del CBCL en su versión para niños de 6 a 11 años en Argentina (Samaniego, 2008), se decidió que sería un aporte valioso, tanto para la actividad clínica como investigativa, estandarizar la versión para preescolares del CBCL. Por lo tanto, se definió el objetivo de establecer la confiabilidad y la validez del CBCL $1 \frac{1}{2}-5$ en lo que respecta a los problemas comportamentales y emocionales, en su versión en español para padres. 


\section{Método}

Participantes

En el presente estudio metodológico se ha empleado un muestreo de tipo no probabilístico intencional. Se trabajó con dos muestras, una de ellas integrada por niños de población clínica, derivados a servicios de salud mental, y la otra formada por niños escolarizados de población general, con la finalidad de verificar si el CBCL 1 1 $\frac{2}{2}-5$ discriminaba adecuadamente entre estos grupos. La muestra de población general fue relevada en jardines de infantes y en jardines maternales públicos (cinco) y privados (cuatro); ubicados en la ciudad de Mercedes, Provincia de Buenos Aires, y en la Ciudad Autónoma de Buenos Aires, Argentina, durante el período agosto 2011 - octubre 2013. Para acceder a la población clínica de salud mental, se recurrió a servicios de salud mental infantil de dos hospitales públicos de la Ciudad Autónoma de Buenos Aires, Argentina. La recolección de datos se extendió durante el período agosto 2011 - diciembre 2014.

De los 587 casos recabados se excluyeron en total 46 protocolos. El criterio de exclusión para la muestra de población general fue que el niño se encontrara bajo tratamiento psicológico y/o psiquiátrico en ese momento o lo hubiera recibido en los últimos 12 meses. Hubo 4 casos en los que este dato no fue informado y otros 14 en los que el niño estaba/había estado bajo tratamiento. También se excluyeron 11 protocolos por no tener los datos sociodemográficos completos o el consentimiento informado firmado; y otros 7 casos por tener el niño más de 6 años. Por último, siguiendo el criterio sugerido por los autores del CBCL, se excluyeron 10 cuestionarios que tenían más de 8 ítems perdidos.

La muestra final se compuso de 541 casos (ver Tabla 1). En el primer grupo, de población general, $(n=369)$ se observa una distribución proporcional semejante según el sexo del niño (49.6\% varones y $50.4 \%$ mujeres), con una edad promedio de $3.97(\mathrm{DE}=1.16)$. En el grupo clínico de salud mental $(\mathrm{n}=172)$ hay una mayor proporción de varones (66.9\% vs. $33.1 \%$ ), lo cual es característico de las consultas clínicas en esta franja etaria; con una edad promedio de $3.94(\mathrm{DE}=1.02)$. Respecto de quién respondió el cuestionario, en un $83.7 \%$ lo hizo la madre del niño, en un $12.6 \%$ el padre del niño y en un $3.7 \%$ otra persona (abuelo/a, tío/a).

Tabla 1

Distribución correspondiente a las muestras de Población General y de Población Clínica de Salud Mental según variables sociodemográficas.

\begin{tabular}{|c|c|c|c|c|}
\hline Variables sociodemográficas & $\begin{array}{c}\text { Grupo Población } \\
\text { General } \\
n=369\end{array}$ & & $\begin{array}{l}\text { Grupo } \\
\text { Clínico } \\
n=172\end{array}$ & \\
\hline Características del niño & $\%$ & $\mathbf{n}$ & $\%$ & $\mathbf{n}$ \\
\hline Sexo & $\begin{array}{l}49.6 \% \text { varones } \\
50.4 \% \text { mujeres }\end{array}$ & $\begin{array}{l}183 \\
186\end{array}$ & $\begin{array}{l}66.9 \% \text { varones } \\
33.1 \% \text { mujeres }\end{array}$ & $\begin{array}{c}115 \\
57\end{array}$ \\
\hline Edad del niño & $\begin{array}{l}\mathrm{M}=3.97, \mathrm{DE}=1.16 \\
\text { Mín }=1.1 ; \text { Máx }=5.9\end{array}$ & & $\begin{array}{l}\mathrm{M}=3.94, \mathrm{DE}=1.02 \\
\text { Mín }=2 ; \text { Máx }=5.9\end{array}$ & \\
\hline \multicolumn{5}{|l|}{ Escolaridad } \\
\hline no concurre a jardín & $1.4 \%$ & 5 & $24.4 \%$ & 42 \\
\hline maternal o guardería & $20.3 \%$ & 75 & $4.1 \%$ & 7 \\
\hline jardín para niños con discapacidad & $0 \%$ & 0 & $2.9 \%$ & 5 \\
\hline jardín de infantes & $78.3 \%$ & 289 & $68.6 \%$ & 118 \\
\hline
\end{tabular}




\begin{tabular}{|c|c|c|c|c|}
\hline Variables sociodemográficas & $\begin{array}{c}\text { Grupo Población } \\
\text { General } \\
n=369\end{array}$ & & $\begin{array}{l}\text { Grupo } \\
\text { Clínico } \\
n=172\end{array}$ & \\
\hline Características del niño & $\%$ & $\mathbf{n}$ & $\%$ & $\mathbf{n}$ \\
\hline \multicolumn{5}{|l|}{ Estado civil de los padres } \\
\hline casados - viviendo juntos & $81 \%$ & 298 & $66.7 \%$ & 114 \\
\hline separados - divorciados & $13.9 \%$ & 51 & $19.3 \%$ & 33 \\
\hline madre sola & $4.3 \%$ & 6 & $11.6 \%$ & 19 \\
\hline padre solo & $0.3 \%$ & 1 & $0.6 \%$ & 1 \\
\hline viudo(a) & $0.3 \%$ & 1 & $1.8 \%$ & 3 \\
\hline nueva pareja & $0.6 \%$ & 2 & $0.6 \%$ & 1 \\
\hline \multicolumn{5}{|l|}{ Máx. nivel de instrucción: madre } \\
\hline hasta secundario incompleto & $28.5 \%$ & 105 & $44.8 \%$ & 77 \\
\hline hasta terciario completo & $45.8 \%$ & 168 & $44.2 \%$ & 76 \\
\hline universitario incompleto - completo & $25.4 \%$ & 93 & $9.9 \%$ & 17 \\
\hline no sabe- no contesta & $0.8 \%$ & 3 & $1.2 \%$ & 2 \\
\hline \multicolumn{5}{|l|}{ Máx. nivel de instrucción: padre } \\
\hline hasta secundario incompleto & $36 \%$ & 127 & $46.4 \%$ & 80 \\
\hline hasta terciario completo & $38.8 \%$ & 137 & $39 \%$ & 67 \\
\hline universitario incompleto-completo & $20.4 \%$ & 72 & $5.2 \%$ & 9 \\
\hline no sabe-no contesta & $4.8 \%$ & 33 & $9.4 \%$ & 16 \\
\hline \multicolumn{5}{|l|}{ Principal sostén económico del hogar } \\
\hline Persona con mayores ingresos & $\begin{array}{l}56.6 \% \text { padre } \\
12.2 \% \text { madre }\end{array}$ & $\begin{array}{c}209 \\
45\end{array}$ & $\begin{array}{l}56.4 \% \text { padre } \\
14.5 \% \text { madre }\end{array}$ & $\begin{array}{l}97 \\
25\end{array}$ \\
\hline Laboralmente activo & $96.2 \%$ & 331 & $90.1 \%$ & 154 \\
\hline Cobertura médica & $\begin{array}{c}79 \% \text { si } \\
20.1 \% \text { no }\end{array}$ & $\begin{array}{c}256 \\
65\end{array}$ & $\begin{array}{l}45.1 \% \text { si } \\
53.1 \% \text { no }\end{array}$ & $\begin{array}{l}73 \\
86\end{array}$ \\
\hline
\end{tabular}

\section{Instrumentos}

Se diseñó y se administró un protocolo que incluía un consentimiento informado; preguntas sobre aspectos sociodemográficos que incluían situación laboral de ambos padres, nivel educativo, estado civil y cantidad de hijos en la familia, entre otros aspectos tomados del cuestionario desarrollado por la Asociación Argentina de Marketing para la evaluación del nivel socioeconómico (AAM, 2010), y el cuestionario Child Behavior Checklist (CBCL 1 1/2-5; Achenbach \& Rescorla, 2000).

El Child Behavior Checklist es un instrumento estandarizado que contiene 99 ítems, a partir de los cuales se pueden obtener distintas medidas de problemas comportamentales y emocionales. Los síndromes que componen las diferentes escalas fueron determinados por los autores mediante un criterio estadístico, identificando cuáles son los problemas que tienden a ocurrir de manera conjunta. Para este cuestionario se describieron tres escalas generales y siete específicas. Una de las escalas generales es la de Problemas Internalizantes, comprende problemas que principalmente ocurren dentro del self y agrupa a las escalas específicas: emocionalmente reactivo, ansioso/depresivo, quejas somáticas y retraimiento. La otra escala amplia, de Problemas Externalizantes, principalmen- 
te se refiere a conflictos que se dan con otras personas y a las expectativas que existen sobre el niño; agrupa a las escalas específicas: problemas de atención y comportamiento agresivo. La escala específica de problemas de sueño no se incluye dentro de ninguna de las anteriores, pero se suma para la tercera escala general que es la de problemas totales. Dado que esta estructura de factores ha sido comprobada en más de 24 sociedades, y por recomendación de los autores, que no lo consideraron necesario, en la presente investigación no se realizó un análisis factorial (Achenbach \& Rescorla, 2000; Ivanova et al., 2010; Rescorla et al., 2011).

El cuestionario puede ser respondido por el padre, madre, tutor, o algún otro adulto que vea al niño en su entorno familiar. La consigna del cuestionario indica a la persona que lea una lista de 99 problemas y pensando en los últimos 2 meses del niño señale en cada ítem: 0 si la frase no es cierta para el niño según lo que la persona sabe; 1 si es cierto algunas veces o de alguna manera, y 2 si es muy cierto u ocurre muy a menudo. En algunos ítems se le pide que describa ese problema, por ejemplo en el ítem 31 , Come o bebe cosas que no son comida. Además, al final de la lista de problemas se presenta el ítem 100 en el que puede agregar cualquier otro problema que el niño tenga y que no se haya mencionado previamente en la lista. Por último, el cuestionario incluye tres preguntas abiertas: ¿Sufre el/la niño(a) de alguna enfermedad, o discapacidad física o mental?; ¿Qué es lo que más le preocupa con respecto del niño(a)? y ¿Qué es lo mejor que ve en el/la niño(a)?

En lo que respecta a la interpretación de los valores que pueden obtenerse a través de este instrumento, puede decirse que cuanto mayor es el puntaje en una subescala esto implica mayor presencia de ese síndrome en el niño según la perspectiva del adulto que responde. Los autores determinaron que un nivel clínico de problemas en las escalas generales (puntaje total, internalizantes y externalizantes) se con- sidera a partir del percentil 90, mientras que un nivel pre-clínico se corresponde con percentiles entre 83 y 90 . En el caso de las escalas específicas (emocionalmente reactivo, ansioso/depresivo, quejas somáticas, retraimiento, problemas de atención, comportamiento agresivo y problemas de sueño) el nivel clínico se considera a partir del percentil 98, y un nivel pre-clínico entre los percentiles 93 y 97 (Achenbach \& Rescorla, 2000).

Los autores del instrumento estudiaron sus propiedades psicométricas examinando diferentes medidas de confiabilidad y validez. Dentro de los estudios de confiabilidad, analizaron mediante el método de test-retest la correlación de las puntuaciones pasado un período de 8 días, el promedio de los niveles de $r$ a través de todas las escalas fue de .85, en un rango de .68 a .92 . Asimismo, en el análisis de la estabilidad de las puntuaciones pasados 12 meses, el promedio de $r$ a través de todas las escalas fue de .61, en un rango de .53 a .76. También se calculó el grado de acuerdo entre informantes, en promedio el coeficiente $r$ fue de .61 a través de todas las escalas, con un rango entre .48 y .67. Respecto de la validez de criterio, las escalas definidas para los distintos problemas discriminaron adecuadamente entre el grupo clínico de salud mental y el de población general. Los autores también analizaron su validez de constructo y su estructura factorial (Achenbach \& Rescorla, 2000).

La versión empleada en este estudio implicó una adaptación lingüística, para la cual se trabajó en colaboración con los autores del CBCL 1 1 1/2-5, Achenbach y Rescorla, Investigadores del Departamento de Psiquiatría de la Universidad de Vermont, referentes internacionales en el área de Psicopatología Infantil. Se revisaron aspectos de equivalencia de contenido, equivalencia semántica y validez de contenido. Se hicieron estudios piloto en una muestra de población general y en una muestra pequeña de población clínica a fin de ajustar la redacción de los ítems, corroborando que los mismos fue- 
ran comprendidos por la población destinataria.

Los cambios que se realizaron fueron: Ítem 7, se modificó "No tolera que las cosas están fuera de lugar" por No tolera que las cosas estén fuera de lugar. Ítem 9, se modificó "Mastica lo que no es comestible" por Muerde cosas que no son comestibles, y se agregó por Ej. la manga. Ítem 12, se modificó "Estreñido(a) no defeca (cuando no está enfermo[a])" por $\mathrm{Pa}$ dece de estreñimiento (cuando no está enfermo[a]). Ítem 19, se modificó "Tiene diarreas o heces líquidas (cuando no está enfermo[a])" por Tiene diarrea (cuando no está enfermo[a]). Ítem 31, se modificó "Come o bebe cosas que no son alimento-no incluya dulces (describa)" por Come o bebe cosas que no son comida-no incluya dulces/golosinas (describa). Ítem 35, se modificó "Pelea mucho" por Se mete en muchas peleas. Ítem 75, se modificó "Se unta o juega con excremento" por Embarra o juega con excremento (caca). Ítem 79, se modificó "Súbitos cambios de tristeza a excitación" por Súbitos/ Bruscos cambios de tristeza a excitación. Ítem 82, se modificó "Súbitos cambios de humor o sentimientos" por Súbitos/Bruscos cambios de humor o sentimientos. Ítem 85, se modificó "Le dan rabietas o tiene mal genio" por Tiene berrinches o mal genio. Ítem 88, se modificó "Poco cooperador(a)" por Poco Colaborador. Por último se cambió la redacción de una de las últimas preguntas "¿Sufre su hijo(a) de alguna enfermedad, o incapacidad física o mental?" por ¿Sufre su hijo(a) de alguna enfermedad, o discapacidad física o mental?

\section{Procedimiento}

Los cuestionarios fueron administrados a los padres y/o madres o al cuidador principal del niño/a. En el caso del grupo de población general se acordaron varios encuentros en las instituciones educativas, las tomas se realizaban de manera autoadministrada y colectiva. En el caso del grupo clínico de salud mental se concurría semanalmente a los servicios de los hospitales públicos, y se invitaba a los padres que acudían a una entrevista de admisión a completar el cuestionario en el momento previo a dicha entrevista. En todos los casos la participación fue anónima y voluntaria, e incluyó la firma de un consentimiento informado.

\section{Tratamiento estadístico de los datos}

Los datos fueron procesados mediante el programa SPSS v. 21. (IBM, 2012). La confiabilidad del instrumento se analizó mediante diferentes métodos:

1. El nivel de consistencia interna se estudió mediante el cálculo de los coeficientes Alfa de Cronbach; considerando índices excelentes a los coeficientes superiores a .90 ; buenos a los superiores a .80 , y aceptables a los superiores a .70 (George \& Mallery, 2001).

2. El grado de acuerdo entre padres se midió a través del coeficiente de correlación $r$ de Pearson; interpretando como niveles de relación muy fuerte a los coeficientes superiores a .90; considerable a los superiores a .75 , y medio a los superiores a .50 (Hernández-Sampieri, Fernández-Collado, \& Baptista-Lucio, 2006).

3. El nivel de cambio en las puntuaciones se estudió mediante el método test-retest entrevistando a un grupo de padres luego de 7 días (corto plazo) y a otro grupo de padres luego de 6 meses (estabilidad). De este modo se calcularon los coeficientes de correlación $r$ de Pearson; interpretando como niveles de relación muy fuerte a los coeficientes superiores a .90 , considerable a los superiores a .75, y medio a los superiores a .50 (Hernández-Sampieri et al., 2006).

El análisis de validez del instrumento se llevó adelante mediante la técnica de grupos contrastados, utilizando la prueba $t$ de Student. Se valoró el tamaño del efecto de las diferencias de medias a partir del cálculo de la $d$ de Cohen; 
interpretando como un tamaño del efecto grande a los valores superiores a .80 , mediano a los superiores a .50 y pequeño a los superiores a .20 (Bologna, 2015; Cárdenas-Castro \& Arancibia-Martini, 2014).

\section{Resultados}

Puntajes brutos para determinar rangos clínico y pre-clínico en población general

Considerando los puntajes de corte propuestos por los autores (Achenbach \& Rescorla, 2000), se determinó para población urbana de Argentina que los puntajes totales del CBCL $1 \frac{1}{2}-5$ iguales o superiores a 67 indican un ni- vel clínico de problemas comportamentales y emocionales (percentil 90); mientras que puntajes entre 58.10 y 67 indican un nivel pre-clínico (percentil 83 a 90). Específicamente para los problemas externalizantes el nivel clínico es considerado a partir de un puntaje igual o superior a 27 (percentil 90) y el nivel pre-clínico se vincula a los puntajes entre 23 y 27 (percentil 83 a 90). Para los problemas de tipo internalizante, el nivel clínico es considerado a partir de un puntaje igual o superior a 20 (percentil 90) y el nivel pre-clínico se vincula a los puntajes entre 18 y 20 (percentil 83 a 90). En la Tabla 2 puede verse el detalle de las puntuaciones que determinan niveles clínicos (percentil 98) y pre-clínicos (percentil 93 a 97) para cada una de las escalas específicas.

Tabla 2

Análisis descriptivo de las puntuaciones de las escalas generales y específicas del CBCL 11/2-5 en población general.

\begin{tabular}{|c|c|c|c|c|c|c|c|c|c|c|}
\hline & $\begin{array}{c}\text { Nivel } \\
\text { Total de } \\
\text { Problemas }\end{array}$ & $\begin{array}{l}\text { Prob. } \\
\text { Ext. }\end{array}$ & $\begin{array}{c}\text { Prob. } \\
\text { Int. }\end{array}$ & $\begin{array}{c}\text { Comportam. } \\
\text { Agresivo }\end{array}$ & $\begin{array}{l}\text { Prob. } \\
\text { de } \\
\text { Atención }\end{array}$ & $\begin{array}{c}\text { Emocional- } \\
\text { mente } \\
\text { Reactivo }\end{array}$ & $\begin{array}{l}\text { Retrai- } \\
\text { miento }\end{array}$ & $\begin{array}{l}\text { Ansioso - } \\
\text { Depresivo }\end{array}$ & $\begin{array}{l}\text { Quejas } \\
\text { Somáticas }\end{array}$ & $\begin{array}{c}\text { Prob. } \\
\text { de } \\
\text { Sueño }\end{array}$ \\
\hline Puntaje mínimo & 3 & 0 & 0 & 0 & 0 & 0 & 0 & 0 & 0 & 0 \\
\hline Puntaje máximo & 90 & 41 & 33 & 36 & 7 & 11 & 8 & 14 & 9 & 14 \\
\hline Media & 39.68 & 15.73 & 10.60 & 12.82 & 2.91 & 2.30 & 1.86 & 4.19 & 2.24 & 2.68 \\
\hline Desvío estándar & 18.86 & 7.78 & 6.52 & 6.52 & 1.76 & 2.17 & 1.86 & 2.47 & 1.94 & 2.17 \\
\hline Percentil 83 & 58.10 & 23 & 18 & & & & & & & \\
\hline Percentil 90 & 67 & 27 & 20 & & & & & & & \\
\hline Percentil 93 & & & & 23.10 & 6 & 6 & 5 & 8 & 5 & 6 \\
\hline Percentil 97 & & & & 27 & 6 & 7.90 & 6 & 9 & 6.90 & 7 \\
\hline Percentil 98 & & & & 28 & 6 & 9 & 7 & 10 & 7 & 8 \\
\hline
\end{tabular}

Niveles de confiabilidad del CBCL 11/2-5

a) Se analizó la consistencia interna del instrumento mediante el cálculo de los coeficientes Alfa de Cronbach, sobre una muestra de 369 casos de población general (ver Tabla 3). Se observaron mejores índices para el puntaje total del cuestionario con un coeficiente superior a $.90(\alpha=.929)$, y para las escalas generales de problemas externalizantes $(\alpha=.892)$ y problemas internalizantes $(\alpha=.816)$, con coeficientes superiores a .80 . Mientras tanto, para las escalas específicas se obtuvieron valores entre $\alpha=.447$ y $\alpha=.879$. 
Tabla 3

Niveles de consistencia interna del CBCL 1 1/2-5, evaluados con el coeficiente Alfa de Cronbach, en población general.

\begin{tabular}{lcc}
\hline & $\alpha$ & $\begin{array}{c}\mathbf{n}^{\mathbf{0}} \text { de } \\
\text { ítem }\end{array}$ \\
\hline Nivel Total de Problemas & .929 & 100 \\
Problemas Externalizantes & .892 & 24 \\
Problemas Internalizantes & .816 & 36 \\
Comportamiento Agresivo & .879 & 19 \\
Problemas de Atención & .577 & 5 \\
Emocionalmente Reactivo & .624 & 9 \\
Retraimiento & .600 & 8 \\
Ansioso Depresivo & .614 & 8 \\
Quejas Somáticas & .447 & 11 \\
Problemas de Sueño & .610 & 7 \\
\hline
\end{tabular}

Nota. $n=369$.

b) Se buscó estimar el grado de acuerdo entre padres y madres, en una porción de la muestra de población general $(\mathrm{n}=88)$. Se utilizó el coeficiente de correlación $r$ de Pearson y se obtuvieron valores superiores a .50 para el puntaje total del instrumento $(r=.634)$, para la escala general de problemas externalizantes $(r$ $=.713)$ y para la escala general de problemas internalizantes $(r=.577)$. Para las escalas específicas se obtuvieron valores entre $r=.437 \mathrm{y}$ $r=.701$. En todos los casos se obtuvo un nivel de significación adecuado ( $p \leq .01$; ver Tabla 4$)$.
Tabla 4

Niveles de confiabilidad del CBCL 1 1/2-5, evaluados por acuerdo entre madre y padre, en población general.

\begin{tabular}{lc}
\hline \multicolumn{1}{c}{ Escala } & $\boldsymbol{r}$ \\
\hline Nivel Total de Problemas & $.634^{* *}$ \\
Problemas Externalizantes & $.713^{* *}$ \\
Problemas Internalizantes & $.577^{* *}$ \\
Comportamiento Agresivo & $.701^{* *}$ \\
Problemas de Atención & $.623^{* *}$ \\
Emocionalmente Reactivo & $.545^{* *}$ \\
Retraimiento & $.478^{* *}$ \\
Ansioso Depresivo & $.581^{* *}$ \\
Quejas Somáticas & $.437 * *$ \\
Problemas de Sueño & $.544^{* *}$ \\
\hline
\end{tabular}

Nota. $* \mathrm{p} \leq .05 ; * * \mathrm{p} \leq .01 ; \mathrm{n}=88$.

c) También se consideró como otra medida de confiabilidad el nivel de cambio en las puntuaciones mediante el método test-retest. En el análisis a corto plazo, luego de 7 días $(\mathrm{n}=94)$, los valores de correlación fueron considerables, con coeficientes superiores a .75. Esto se observó para el puntaje total $(r=.887)$, y para las escalas generales de problemas externalizantes $(r=.892)$ e internalizantes $(r=.848)$. En el caso de las escalas específicas, los coeficientes de correlación variaron entre $r=.697$ y $r=.891$. Las escalas que tuvieron coeficientes menores fueron las de problemas de sueño, retraimiento y problemas de atención. En todos los casos se obtuvo un nivel de significación adecuado ( $p \leq$ .01; ver Tabla 5). En el análisis de estabilidad, luego de 6 meses $(n=111)$, los valores de correlación fueron medios, con coeficientes superiores a .50. Esto se observó para el puntaje total $(r=.630)$, las escalas generales de problemas externalizantes $(r=.633)$ y problemas internalizantes $(r=.681)$ y todas las escalas específicas a excepción de la escala de quejas somáticas ( $r$ $=.482$ ). En todos los casos se obtuvo un nivel de significación adecuado ( $p \leq .01$; ver Tabla 5$)$. 
Tabla 5

Niveles de confiabilidad y estabilidad de los puntajes del CBCL 11/2-5, en población general.

Confiabilidad test-retest

\begin{tabular}{lcc}
\hline & 7 -días r & 6 -meses r \\
& $\mathrm{n}=94=111$ \\
Nivel Total de Problemas & $.887 * *$ & $.630^{* *}$ \\
Problemas Externalizantes & $.892^{* *}$ & $.633^{* *}$ \\
Problemas Internalizantes & $.848^{* *}$ & $.681^{* *}$ \\
Comportamiento Agresivo & $.891^{* *}$ & $.641^{* *}$ \\
Problemas de Atención & $.697^{* *}$ & $.546^{* *}$ \\
Emocionalmente Reactivo & $.750^{* *}$ & $.575^{* *}$ \\
Retraimiento & $.735^{* *}$ & $.642^{* *}$ \\
Ansioso Depresivo & $.772^{* *}$ & $.611^{* *}$ \\
Quejas Somáticas & $.785^{* *}$ & $.482^{* *}$ \\
Problemas de Sueño & $.743^{* *}$ & $.642^{* *}$ \\
\hline
\end{tabular}

El método elegido en este estudio para analizar la validez del cuestionario fue el de grupos contrastados, para ello se examinó el poder de discriminación del instrumento entre un grupo clínico de salud mental (niños que eran llevados por sus padres a una primera entrevista de admisión) y un grupo de comparación de niños de la población general (que no se encontraban en tratamiento psicológico o psiquiátrico en ese momento ni lo habían recibido en los últimos 12 meses). Se informan a continuación los puntajes promedio para cada una de las escalas generales y específicas, las diferencias de medias por la prueba $t$ de Student y los tamaños del efecto a partir del cálculo de la $d$ de Cohen. Los mismos fueron calculados separadamente para varones y mujeres (ver Tabla 6).

\section{Tabla 6}

Niveles de validez del CBCL 1 1 $12-5$, evaluados por el poder de discriminación del instrumento entre un grupo clínico de salud mental y un grupo de población general.

\begin{tabular}{|c|c|c|c|c|c|c|c|c|c|c|c|c|c|c|}
\hline \multirow[b]{3}{*}{$\begin{array}{l}\text { Escalas del } \\
\text { CBCL } \\
\end{array}$} & \multicolumn{4}{|c|}{ Varones } & \multicolumn{10}{|c|}{ Mujeres } \\
\hline & \multicolumn{2}{|c|}{$\begin{array}{c}\text { Grupo } \\
\text { Pob. Gral } \\
n=183\end{array}$} & \multicolumn{2}{|c|}{$\begin{array}{c}\text { Grupo } \\
\text { Clínico } \\
n=115\end{array}$} & \multirow[b]{2}{*}{$p$} & \multirow[b]{2}{*}{$d$} & \multicolumn{3}{|c|}{$\begin{array}{c}\text { Grupo } \\
\text { Pob. Gral } \\
n=186\end{array}$} & \multicolumn{2}{|c|}{$\begin{array}{l}\text { Grupo } \\
\text { Clínico } \\
n=57\end{array}$} & \multirow[b]{2}{*}{$p$} & \multirow[b]{2}{*}{$d$} & \multirow[b]{2}{*}{$r$} \\
\hline & Media & DE & Media & DE & & & $r$ & Media & DE & Media & DE & & & \\
\hline Nivel Total de Problemas & 41.75 & 18.71 & 65.48 & 26.20 & .000 & 1.04 & .46 & 37.65 & 18.85 & 54.14 & 28.11 & .000 & .68 & .32 \\
\hline Problemas Externalizantes & 16.86 & 7.91 & 23.71 & 9.34 & .000 & .79 & .36 & 14.62 & 7.51 & 18.51 & 10.61 & .012 & .42 & .20 \\
\hline Problemas Internalizantes & 10.96 & 6.73 & 19.79 & 10.29 & .000 & 1.01 & .45 & 10.24 & 6.32 & 16.45 & 10.47 & .000 & .71 & .33 \\
\hline Comportam. Agresivo & 13.67 & 6.70 & 18.67 & 8.05 & .000 & .67 & .31 & 11.99 & 6.26 & 14.46 & 9.18 & .062 & .31 & .15 \\
\hline Problemas de Atención & 3.19 & 1.74 & 5.04 & 1.98 & .000 & .99 & .44 & 2.63 & 1.75 & 4.05 & 2.16 & .000 & .72 & .33 \\
\hline
\end{tabular}




\begin{tabular}{|c|c|c|c|c|c|c|c|c|c|c|c|c|c|c|}
\hline \multirow[b]{3}{*}{$\begin{array}{c}\text { Escalas del } \\
\text { CBCL }\end{array}$} & \multicolumn{4}{|c|}{ Varones } & \multicolumn{10}{|c|}{ Mujeres } \\
\hline & \multicolumn{2}{|c|}{$\begin{array}{c}\text { Grupo } \\
\text { Pob. Gral } \\
n=183\end{array}$} & \multicolumn{2}{|c|}{$\begin{array}{c}\text { Grupo } \\
\text { Clínico } \\
n=115\end{array}$} & \multirow[b]{2}{*}{$p$} & \multirow[b]{2}{*}{$d$} & \multicolumn{3}{|c|}{$\begin{array}{c}\text { Grupo } \\
\text { Pob. Gral } \\
n=186\end{array}$} & \multicolumn{2}{|c|}{$\begin{array}{l}\text { Grupo } \\
\text { Clínico } \\
n=57\end{array}$} & \multirow[b]{2}{*}{$p$} & \multirow[b]{2}{*}{$d$} & \multirow[b]{2}{*}{$r$} \\
\hline & Media & DE & Media & $\mathbf{D E}$ & & & $r$ & Media & DE & Media & DE & & & \\
\hline Emocional- mente Reactivo & 2.44 & 2.33 & 4.59 & 3.54 & .000 & .71 & .33 & 2.16 & 2.01 & 3.49 & 3.06 & .003 & .51 & 2.24 \\
\hline Retraimiento & 1.95 & 2.01 & 5.34 & 3.57 & .000 & 1.17 & .50 & 1.77 & 1.71 & 3.88 & 3.17 & .000 & .82 & .38 \\
\hline Ansioso Depresivo & 4.28 & 2.56 & 6.48 & 3.23 & .000 & .75 & .35 & 4.11 & 2.39 & 5.70 & 3.14 & .001 & .56 & .27 \\
\hline Quejas Somáticas & 2.28 & 1.85 & 3.38 & 2.83 & .000 & .46 & .22 & 2.20 & 2.03 & 3.35 & 3.58 & .024 & .39 & .19 \\
\hline Problemas de Sueño & 2.52 & 1.87 & 3.97 & 3.00 & .000 & .58 & .27 & 2.63 & 1.75 & 4.05 & 2.16 & .078 & .72 & .33 \\
\hline
\end{tabular}

Nota. Puntajes promedio del Child Behavior Checklist, escalas y total por sexo y situación de derivación, en niños de 1 $1 / 2$ a 5 años.

Al considerar las escalas generales, se encontraron valores más altos en el grupo clínico en comparación con el grupo de población general, tanto para los varones (puntaje total, $\mathrm{M}=$ 65.48 vs. $\mathrm{M}=41.75$; problemas externalizantes, $\mathrm{M}=23.71$ vs. $\mathrm{M}=16.86$; problemas internalizantes, $M=19.76$ vs. $M=10.96$ ), como para las mujeres (puntaje total, $\mathrm{M}=54.14$ vs. $\mathrm{M}=$ 37.65; problemas externalizantes, $\mathrm{M}=18.51$ vs. $M=14.62$; problemas internalizantes, $M=$ 16.45 vs. $M=10.24)$. Estas diferencias de medias resultaron ser estadísticamente significativas según lo verificado mediante una prueba $t$ de Student $(p \leq .05)$, y tener tamaños del efecto entre pequeños y medianos ( $d$ entre .20 y .50$)$; evidenciando un excelente nivel de validez del instrumento. Por otra parte, al revisar en detalle las escalas específicas, los resultados muestran cierta variabilidad entre varones y mujeres. En el caso de los varones todas las escalas específicas tienen diferencias significativas entre las medias $(p \leq .01)$, siendo más altas en el grupo clínico de salud mental en comparación con el grupo de población general, con tamaños del efecto entre pequeños y medianos ( $d$ entre .20 y .50). Mientras tanto, en el caso de las mujeres la mayoría de las escalas específicas muestran diferencias significativas entre las medias $(p \leq .05)$, siendo más altas en el grupo clínico de salud mental en comparación con el grupo de población general, con tamaños del efecto entre pequeños y medianos ( $d$ entre .20 y .50 ). Quedan fuera de estos niveles aceptables las escalas específicas de comportamiento agresivo y problemas de sueño. Esto puede deberse al número de casos más reducido de la muestra de mujeres en el grupo clínico, y requiere de mayores análisis en el futuro.

\section{Discusión}

Debido a que demuestra la confiabilidad y validez del CBCL 1 1 $1 / 2-5$, el presente estudio es un aporte instrumental relevante para el diagnóstico precoz de problemas comportamentales y emocionales en edades tempranas. La disponibilidad de este instrumento permitirá reducir la brecha existente entre las primeras preocupaciones de los padres, pediatras o maestros respecto a la salud mental infantil, y las posibilidades de ofrecer un proceso psicoterapéutico que mejore el pronóstico del niño, y disminuya la aparición de trastornos o discapacidad a largo plazo (Achenbach \& Rescorla, 2000; Martinez, 2009; Pihlakoski et al., 2006). 
Se ha ofrecido información valiosa que complementa los estudios realizados en la región (Lecannelier et al., 2014; Lins \& Alvarenga, 2015; Rivas et al., 2011; Samaniego, 2004, 2012, 2015; Schu et al., 2011; Slapak et al., 2002) buscando superar las limitaciones advertidas en el conocimiento actual respecto de la conceptualización, definición, medición y estudio de los problemas comportamentales y emocionales en niños pequeños de edad preescolar (Dougherty et al., 2015; Egger \& Angold, 2006; Skovgaard et al., 2004; Skovgaard et al., 2008).

En la muestra estudiada para población general urbana de Argentina, la media de problemas totales fue de 39.68, siendo levemente más elevada que lo informado para Estados Unidos pero más baja por ejemplo que lo informado para Chile, y similar a lo informado en otras sociedades como Rumania, Singapur, Irán y Turquía. Considerando el rango (17.2 - 47.5) que se propone en el estudio con 24 sociedades en una muestra de $\mathrm{N}=19,850$ (media omnicultural 33.3), la media de problemas para población Argentina se encuentra dentro del desvío estándar (7.1) que informan los investigadores (Rescorla et al., 2011).

Uno de los resultados más importantes del presente estudio es haber demostrado la validez del instrumento, esto quiere decir que el CBCL $1 \frac{1}{2}-5$ es capaz de evaluar la presencia de distintos grados de problemas comportamentales y emocionales en edades muy tempranas. Las escalas generales (puntaje total, problemas externalizantes y problemas internalizantes) mostraron diferencias entre las medias, siendo más altas en el grupo clínico de salud mental en comparación con el grupo de población general. Estas diferencias resultaron estadísticamente significativas y con tamaños del efecto entre pequeños y medianos. Las escalas específicas (problemas de atención, comportamiento agresivo, emocionalmente reactivo, ansioso/depresivo, quejas somáticas, retraimiento y problemas de sueño) mostraron niveles de validez muy sa- tisfactorios para el grupo de varones. Sin embargo, para el grupo de mujeres, las escalas de comportamientos agresivos y problemas de sueño requieren de mayores estudios, debido a que la proporción de niñas entre los grupos no fue similar. Este mismo procedimiento había sido realizado por los autores del instrumento con resultados satisfactorios, tanto para varones como para mujeres (Achenbach \& Rescorla, 2000).

En cuanto a la confiabilidad del instrumento, en primer lugar, pudo demostrarse un excelente nivel de consistencia interna para el puntaje total, con un coeficiente Alfa superior a .90. También se encontraron buenos niveles de confiabilidad para las escalas generales de problemas internalizantes y externalizantes, con coeficientes superiores a .80. Al considerar las escalas específicas, únicamente se obtuvo un buen índice para la subescala de comportamiento agresivo, con un coeficiente superior a .80 . Mientras tanto, el resto de las escalas específicas no llegaron a tener un índice aceptable, dado que los coeficientes fueron menores a .70. Estas diferencias son semejantes a lo informado en el manual del cuestionario (Achenbach \& Rescorla, 2000) y en un estudio transcultural (Rescorla et al., 2011). Una posible interpretación de los bajos coeficientes encontrados en algunas de las escalas específicas tiene que ver con el número de ítems analizado, en estas escalas la cantidad de ítems considerados en el análisis era menor si se compara con la cantidad de ítems que componen las escalas generales.

Como segunda medida de confiabilidad del instrumento, se obtuvieron niveles altos de acuerdo entre informantes (madre y padre). Se vio un nivel medio de correlación $(r>.50)$ para el puntaje total, escalas generales y todas las escalas específicas, a excepción de quejas somáticas y retraimiento (Hernández-Sampieri, Fernández-Collado, \& Baptista-Lucio, 2006).

Como tercera y última medida de confiabilidad se demostró, mediante el método de 
test-retest, que las puntuaciones son semejantes pasados 7 días y pasados 6 meses. Los valores de correlación del puntaje total del instrumento $\mathrm{y}$ de las escalas generales de problemas internalizantes y externalizantes fueron mejores a los 7 días, con coeficientes $r$ superiores a .75 , respecto de los 6 meses, con coeficientes $r$ superiores a .50. Estos resultados satisfactorios son similares a lo informado por los autores (Achenbach \& Rescorla, 2000).

A modo de conclusión puede decirse lo siguiente:

a) Los niveles de problemas externalizantes e internalizantes de niños y niñas en edad preescolar de Argentina son similares a los encontrados en otros estudios internacionales.

b) Los puntajes de corte para un rango clínico y pre-clínico, identificados en el presente estudio, podrán ser tomados como parámetro en futuras investigaciones empíricas y tendrán valor clínico como indicadores de eficacia del tratamiento.

c) La estandarización del CBCL 1 1 1/2-5 permite contar con un instrumento de detección temprana de problemas comportamentales $\mathrm{y}$ emocionales, fortaleciendo la capacidad diagnóstica de problemas en la salud mental infantil.

\section{Referencias}

Achenbach, T. M. (1992). Manual for the Child Behavior Checklist/2-3. Burlington, VT: University of Vermont, Department of Psychiatry.

Achenbach, T., \& Rescorla, L. (2000). Manual for the Aseba Preschool Forms \& Profiles. Burlington, VT: University of Vermont. Research Center for Children, Youth, \& Families.

Achenbach, T. M., \& Rescorla, L. (julio, 2004). Practical Applications of the Achenbach System of Empirically Based Assessment (ASEBA) for Ages 1.5 to $90+$ Years. Trabajo presentado en la International Test Users' Conference. ACER, Melbourn, Australia. Recuperado de http://research.acer.edu.au/ research conferenceITU 2004/2

Achenbach, T. M., Rescorla, L. A., \& Maruish, M. (2004). The Achenbach System of Empirically Based Assessment (ASEBA) for ages 1.5 to 18 years. En Maruish M. E. (Ed.). The Use of Psychological Testing for Treatment Planning and Outcomes Assessment, 2, 179-213. Londres: Laurence Erlbaum. Asociación Argentina de Marketing. (2010). Índice de Nivel Socioeconómico Argentino, Buenos Aires, Argentina.

Bologna, E. (2015). Estimación por intervalo del tamaño del efecto expresado como proporción de varianza explicada. Revista Evaluar, 14. Recuperado de https://revistas.unc.edu.ar/index.php/revaluar

Cárdenas-Castro, M. C., \& Arancibia-Martini, H. A. (2014). Potencia estadística y cálculo del tamaño del efecto en $\mathrm{G}^{*}$ Power: Complementos a las pruebas de significación estadística y su aplicación en psicología. Salud \& Sociedad, 5(2), 210-224. Recuperado de http://revistas.ucn.cl/index.php/saludysociedad

Cervone, N., Samaniego, C., Luzzi, A. M., Slapak, S., Padawer, M., \& Frylinztein, C. (agosto, 2003). Enfoque epidemiológico en una población clínica de niños escolarizados. Memorias de las X Jornadas de Investigación en Psicología, (pp 25-28). Facultad de Psicología, Universidad de Buenos Aires. ISSN 1667-6750.

Dougherty, L. R., Leppert, K. A., Merwin, S. M., Smith, V. C., Bufferd, S. J., \& Kushner, M. R. (2015). Advances and directions in preschool mental health research. Child Development Perspectives, 9(1), 14-19. doi: 10.1111/cdep.12099

Egger, H. L., \& Angold, A. (2006). Common emotional and behavioral disorders in preschool children: Presentation, nosology, and epidemiology. Journal of Child Psychology and Psychiatry, 47(3-4), 313337. doi: 10.1111/j.1469-7610.2006.01618.x

George, D., \& Mallery, P. (2001). SPSS for Windows step by step: A simple guide and reference. Boston, MA: Allyn and Bacon.

Hernández-Sampieri, R., Fernández-Collado, C., \& Baptista-Lucio, P. (2006). Metodología de la Investigación. (4 ${ }^{\circ}$ ed.). México: McGraw-Hill. 
IBM (2012). SPSS Statistics 21.0.0. [software de cómputo]. Recuperado de https://www-01.ibm.com/ software/mx/analytics/spss/products/statistics

Ivanova, M. Y., Achenbach, T. M., Rescorla, L. A., Harder, V. S., Ang, R. P., Bilenberg, N., . . Verhulst, F. C. (2010). Preschool psychopathology reported by parents in 23 societies: Testing the seven-syndrome model of the Child Behavior Checklist for ages 1.5-5. Journal of the American Academy of Child \& Adolescent Psychiatry, 49(12), 1215-1224. doi: 10.1016/j.jaac.2010.08.019

Lecannelier, F., Pérez-Ewert, J. C., Groissman, S., Gallardo, D., Bardet, A. M., Bascuñan, A., \& Rodríguez, J. (2014). Validation of Child Behavior Inventory for Children ages $1 \frac{1}{2}$ to 5 years (CBCL $1 \frac{1}{2}-5$ ) at the Santiago de Chile City. Universitas Psychologica, 13(2), 491-500. doi: 10.11144/Javeriana. UPSY13-2.vici

Limberg, K., Gruber, K., \& Noterdaeme, M. (2016). The German version of the Child Behavior Checklist 1.5-5 to identify children with a risk of autism spectrum disorder. Autism: The International Journal of Research and Practice, 21(3), 368-374. doi: 10.1177/1362361316645932

Lins, T., \& Alvarenga, P. (2015). Controle psicológico materno e problemas internalizantes em pré-escolares. Psicologia: Teoria e Pesquisa, 31(3), 311319. doi:10.1590/0102-37722015032092311319

Liu, J., Leung, P. W., McCauley, L., Ai, Y., \& Pinto-Martin, J. (2013). Mother's environmental tobacco smoke exposure during pregnancy and externalizing behavior problems in children. Neurotoxicology, 34, 167-174. doi: 10.1016/j.neuro.2012.11.005

Liu, J., Leung, P., Sun, R., Li, H.-T., \& Liu, J.-M. (2012). Cross-cultural application of Achenbach System of Empirically Based Assessment: Instrument translation in Chinese, challenges, and future directions. World Journal of Pediatrics, 8(1), 5-10. doi: 10.1007/s12519-011-0329-X

Liu, J., Liu, X., Wang, W., McCauley, L., Pinto-Martin, J., Wang, Y., ... Rogan, W. J. (2014). Blood lead concentrations and children's behavioral and emotional problems. JAMA Pediatrics, 168(8), 737. doi: 10.1001/jamapediatrics.2014.332
Martinez, C. (2009). Salud Mental en la infancia y adolescencia. En Organización Panamericana de la Salud, Salud Mental en la Comunidad, (pp. 231244). Washington, D.C.: Serie PALTEX.

Molina, M. F., Calero, A., \& Raimundi, M. J. (2014). Escala de Evaluación del Comportamiento del Niño en su versión para Padres (PRS): Adaptación para su uso en Buenos Aires. Revista Evaluar, 13. Recuperado de https://revistas.unc.edu.ar/index.php/ revaluar

Müller, J. M., Averbeck-Holocher, M., Romer, G., Fürniss, T., Achtergarde, S., \& Postert, C. (2015). Psychiatric treatment outcomes of preschool children in a family day hospital. Child Psychiatry \& Human Development, 46(2), 257-269. doi: 10.1007/ s10578-014-0465-3

Muratori, F., Narzisi, A., Tancredi, R., Cosenza, A., Calugi, S., Saviozzi, I., ... Calderoni, S. (2011). The CBCL 1.5-5 and the identification of preschoolers with autism in Italy. Epidemiology and Psychiatric Sciences, 20(04), 329-338. doi: 10.1017/ s204579601100045x

Pandolfi, V., Magyar, C. I., \& Dill, C. A. (2009). Confirmatory factor analysis of the Child Behavior Checklist 1.5-5 in a sample of children with autism spectrum disorders. Journal of Autism and Developmental Disorders, 39(7), 986-995. doi: 10.1007/ s10803-009-0716-5

Pihlakoski, L., Sourander, A., Aromaa, M., Rautava, P., Helenius, H., \& Sillanpää, M. (2006). The continuity of psychopathology from early childhood to preadolescence. European Child \& Adolescent Psychiatry, 15(7), 409-417. doi: 10.1007/s00787006-0548-1

Predescu, E., Şipos, R., Dobrean, A., \& Miclutia, I. (2013). The discriminative power of the CBCL 1.5-5 between autism spectrum disorders and other psychiatric disorders. Journal of Cognitive \& Behavioral Psychotherapies, 13(1), 75-87. ISSN: 2068-7621

Rescorla, L. A. (2005). Assessment of young children using the Achenbach System of Empirically Based Assessment (ASEBA). Mental Retardation and Developmental Disabilities Research Reviews, 
11(3), 226-237. doi: 10.1002/mrdd.20071

Rescorla, L. A., Achenbach, T. M., Ivanova, M. Y., Harder, V. S., Otten, L., Bilenberg, N., ... Verhulst, F. C. (2011). International comparisons of behavioral and emotional problems in preschool children: $\mathrm{Pa}-$ rents' reports from 24 societies. Journal of Clinical Child \& Adolescent Psychology, 40(3), 456-467. doi: 10.1080/15374416.2011.563472

Rivas, M. S., Vázquez, N., \& Samaniego, V. C. (2011). La salud mental de los más pequeños, su relación con la salud mental de sus padres. Memorias de las XVIII Jornadas de Investigación en Psicología. Facultad de Psicología, Universidad de Buenos Aires. Recuperado de http://jimemorias.psi.uba.ar Samaniego, V. C. (2004). Prevalencia de trastornos psíquicos en población escolar de 6 a 11 años de edad. Memorias de las XI Jornadas de Investigación en Psicología. Facultad de Psicología, Universidad de Buenos Aires. Recuperado de http://jimemorias. psi.uba.ar

Samaniego, V. C. (2005) Problemas comportamentales y sucesos de vida en niños de 6 a 11 años de edad. Revista Psykhe, 14(2), 97-108. doi: 10.4067/ s0718-22282005000200008

Samaniego, V. C. (2008). El Child Behavior Checklist: $\mathrm{Su}$ estandarización en población urbana argentina. Revista de Psicología Universidad Católica Argentina, 4(8), 113-130. Recuperado de http:// www.uca.edu.ar/index.php/site/index/es/uca/facultad-de-psicologia-y-psicopedagogia/publicaciones/revista-de-psicologia

Samaniego, V. C. (2012). Problemas comportamentales y emocionales y tolerancia parental en niños pequeños, ¿estabilidad o cambio? Memorias de las XIX Jornadas de Investigación en Psicología. Facultad de Psicología, Universidad de Buenos Aires. Recuperado de http://jimemorias.psi.uba.ar

Samaniego, V.C. (2015). Factores asociados a la Salud Mental Infantil: Diferencias entre padres y madres en sus interacciones con los niños. Memorias de las XXII Jornadas de Investigación en Psicología. Facultad de Psicología, Universidad de Buenos Aires. Recuperado de http://jimemorias.psi.uba.ar Schu, M., Zan, \& Vázquez, N. (junio, 2011). Motivos de consulta infanto-juveniles: Diferencias según variables sociodemográficas. Trabajo presentado en el XVIII Congreso Latinoamericano de FLAPIA y XV Congreso de AAPI. Buenos Aires, Argentina.

Skovgaard, A., Houmann, T., Landorph, S., \& Christiansen, E. (2004). Assessment and classification of psychopathology in epidemiological research of children 0-3 years of age. European Child \& Adolescent Psychiatry, 13(6), 337-346. doi: 10.1007/ s00787-004-0393-z

Skovgaard, A. M., Olsen, E. M., Christiansen, E., Houmann, T., Landorph, S., \& Jørgensen, T. (2008). Predictors (0-10 months) of psychopathology at age $1 \frac{1}{2}$ years-a general population study in The Copenhagen Child Cohort CCC 2000*. Journal of Child Psychology and Psychiatry, 49(5), 553-562. doi: 10.1111/j.1469-7610.2007.01860.x

Slapak, S., Cervone, N., Luzzi, A. M., \& Samaniego, C. (2002). Aplicación del enfoque epidemiológico a una población clínica de niños. Psico-USF, 7(1), $67-76$. 